Rev. Elev. Méd, vét. Pays trop., 1972, 25 (1) : 73-77

\title{
La cysticercose bovine au Tchad Essai de diagnostic sérologique
}

\author{
par C. MARTIN (*)
}

\begin{abstract}
RESUME
Au Tchad, le diagnostic sérologique de la cysticercose bovine est difficile, en raison des réactions croisées qu'entraîne, chez le zébu, l'existence d'un polyparasitisme abondant.

Les méthodes manquent, en général, de spécificité. Les meilleures sont l'agglutination du latex et l'hémagglutination passive.
\end{abstract}

Le travail réalisé au Laboratoire de Farcha a consisté en l'étude de quelques-unes des réactions sérologiques permettant le diagnostic de la cysticercose du vivant de l'animal.

On conçoit l'intérêt de ce diagnostic au moment du choix des animaux d'élevage : ce serait le meilleur moyen d'éliminer les porteurs de cysticerques et de rentabiliser ainsi une opération industrielle.

\section{L'IMMUNITE DANS LA CYSTICERCOSE BOVINE}

Les cysticerques (Cysticercus bovis dans les cas de cysticercose bovine), logés dans l'intimité des tissus, sont capables de solliciter, de la part de l'organisme qui les héberge, la formation d'anticorps: ils ont donc un pouvoir antigénique propre.

Ils peuvent être non immunigènes, c'est-àdire susceptibles de provoquer la formation d'anticorps, sans favoriser le développement d'un état "d'immunité de protection " chez les sujets infestés, les anticorps élaborés par l'individu n'étant alors que les simples témoins de l'infestation vermineuse. C'est le cas des très

(*) I.E.M.V.T. Laboratoire de Farcha, B.P. n ${ }^{\circ} 433$, Fort-Lamy, Tchad. jeunes animaux qui ne sont pas immunisés, bien que porteurs de cysticerques.

Les antigènes peuvent être également immunigènes, c'est-à-dire capables de déterminer chez l'hôte une réaction immunitaire protectrice qui empêche une nouvelle infestation.

Certains parasites élaborent des antigènes spécifiques, d'autres non spécifiques, ce qui pose un problème pour le diagnostic des parasitoses dans le cas de communautés antigéniques : on observe fréquemment des réactions croisées entre zooparasites d'espèces, de familles et même d'ordre différents. Aussi, est-il parfois difficile, en région d'endémicité, d'évaluer le degré de signification de réactions sérologiques positives à un antigène donné. Ceci a été particulièrement bien étudié par CAPRON et collab. (1968) (2) par la technique de l'immunoélectrophorèse qui révèle, par exemple, 16 antigènes communs à Taenia saginata et à Moniezia expansa.

En matière de cysticercose bovine, l'immunité est essentiellement une immunité acquise à la suite d'infestations primaires; c'est une immunité vraie qui persiste même après la mort des vésicules; elle est solide et durable et s'accompagne d'un taux élevé d'anticorps sériques.

On peut la conférer expérimentalement en administrant à de jeunes animaux des oufs de 
Taenia saginata. GRABER et THOME (1964) (5) font cette expérience sur 22 bouvillons et tirent des conclusions intéressantes sur les conditions d'infestation naturelle des animaux de boucherie dont un tiers seulement est atteint de cysticercose : ce sont ceux qui ont échappé à l'immunisation dans les premiers mois de leur existence.

\section{LES METHODES}

Diverses réactions ont été mises en œuvre au Laboratoire de Farcha en vue d'étudier les possibilités d'un diagnostic sérologique de la cysticercose du vivant de l'animal : précipitation-diffusion en gélose de Oudin-Ouchterlony; fixation du complément (Méthode de Kolmer) d'après PAUTRIZEL et collab. (1960) (14); agglutination des particules de latex, selon la technique mise au point par SINGER et PLOTZ (1956) (18); hémagglutination passive. Dans ce cas, les antigènes protidiques sont fixés sur des hématies au moyen d'un agent de couplage, le glutaraldéhyde et cette méthode a été souvent employée dans le diagnostic de la cysticercose humaine par POWELL et collab. (1966).

Ces différents essais ont porté sur des sérums de bovins et des sérums de lapins hyperimmunisés à l'aide de divers antigènes.

Les sérums de bovins provenaient de la région de Fort-Lamy. Ils ont été récoltés à la faveur de plusieurs enquêtes parasitologiques effectuées entre 1965 et 1968 . Chaque animal a été autopsié et les helminthes présents dans l'organisme ont été soigneusement recueillis, comptés et déterminés.

Nous avons pu ainsi disposer :

- de sérums d'animaux indemnes de cysticercose (animaux sains ou animaux porteurs des parasites autres que des cysticerques);

- de sérums d'animaux atteints de ladrerie et, bien souvent, d'autres parasitoses internes. 58 sérums de bovins ont pu être éprouvés. Ces animaux hébergeaient un nombre variable de cysticerques:

Un seul cysticerque : 12 bêtes;

Deux ou trois cysticerques : 13 bêtes;

De sept à douze cysticerques : 2 bêtes;

Cysticercose généralisée : 8 bêtes.

- d'immuns sérums provenant de lapins immunisés au moyen d'un antigène délipidé, obtenu selon le protocole suivant: les lapins ont reçu pendant trois semaines, à raison de trois injections par semaine, une quantité croissante de suspension antigènique en intraveineuse. Huit jours plus tard, une injection de rappel est pratiquée. L'immunisation a été effectuée à l'aide de divers antigènes: Cysticercus bovis, Taenia saginata, Fasciola gigantica et Moniezia expansa. Les lapins sont saignés quelques jours après l'injection de rappel.

Le matériel antigénique utilisé au cours de ces essais a été préparé à partir des helminthes dont il vient d'être question. L'antigène le plus fréquemment employé est extrait de cysticerques délipidés, selon une technique mise au point par PAUTRIZEL. On procède ensuite:

- soit à une extraction aqueuse, par reprise du matériel solide avec de l'eau distillée tamponnée à $\mathbf{P h} 7,2$, suivie d'une centrifugation. Le surnageant recueilli qui représente l'antigène doit posséder une teneur en protéine de $10 \mathrm{mg}$ par $\mathrm{ml}$;

- soit à une extraction alcoolique, par action de l'alcool absolu sur le matériel solide à raison de 1 à $10 \mathrm{~g}$ pour $100 \mathrm{ml}$ pendant 24 heures à $37^{\circ} \mathrm{C}$. On filtre et on laisse évaporer sous vide jusqu'à réduction du volume initial (en général un tiers);

- soit à une extraction polysaccharidique, selon la technique de MELCHER et CAMPBELL (1942) (12) qui font agir successivement un tampon acétate à $\mathrm{Ph} 4,6$, du Chlorure de sodium à 0,5 p. 100 et de l'alcool à 95 p. 100.

L'ensemble des sérums recueillis a été testé vis-à-vis des antigènes ainsi obtenus.

\section{RESULTATS}

1. La technique de précipitation-diffusion en gélose, révélant les anticorps précipitants, montre que les communautés antigéniques entre Fasciola gigantica et Cysticercus bovis sont, dans ce cas, un obstacle important au diagnostic spécifique de la cysticercose. En effet, le même nombre de lignes de précipitation a été révélé en utilisant contre un extrait antigènique de Cysticercus bovis, soit un immun sérum de Cysticercus bovis, soit un immun sérum de Fasciola gigantica.

En outre. en Europe, sur des animaux de boucherie moins parasités qu'en Afrique, Ie 
test de diffusion en gélose est insuffisant, car, il ne permet de déceler que 41 p. 100 des animaux atteints de ladrerie (FRICK et SUSSE, 1970) (4).

2. La réaction de fixation du complément a donné des résultats plus intéressants.

2.1. Les immuns sérums à Cysticercus bovis possèdent des anticorps titrant $1 / 64$ et $1 / 128$ vis-à-vis de l'antigène spécifique.

2.2. Trente sept sérums de bovins atteints de cysticercose dûment constatée à l'autopsie ont été examinés. Les taux d'anticorps les plus élevés ont été obtenus chez trois animaux dont deux présentaient une cysticercose généralisée et le dernier une localisation cardiaque à cysticerques vivants.

Dans 80 p. 100 des cas de ladrerie localisée ou généralisée, sans que les parasites aient subi une quelconque dégénérescence, les taux sérologiques étaient de l'ordre du $1 / 4$ et du $1 / 8$. Enfin, des taux nuls ou faibles $(1 / 2)$ ont été observés chez des animaux porteurs de cysticerques calcifiés.

2.3. Mais, on a obtenu des réactions positives avec les anticorps Fasciola et Moniezia, traduisant des réactions croisées dues à la présence d'anticorps induits par les mêmes substances antigéniques existant dans l'organisme des helminthes utilisés. De ce fait, la sensibilité de la réaction de fixation du complément paraît restreinte. Les échecs enregistrés mettent en cause, soit l'antigène, soit le sérum lui-même.

2.4. La déviation du complément a également été étudiée en Allemagne dans le diagnostic de la ladrerie bovine. Le test est positif un mois après l'infestation expérimentale. Vers le troisième mois, les titres sont à des taux très élevés. La réaction se négative vers le sixième mois (LAMINA et HEIN, 1970) (9), bien que des cysticerques vivants soient visibles, à l'autopsie, dans les muscles de l'animal, ce qui enlève toute valeur pratique à la méthode.

Par ailleurs, de fausses réactions positives sont possibles dans 15 p. 100 des cas (FRICK et SÜSSE, 1970).

3. La réaction d'agglutination du latex est plus significative :

3.1. Tous les sérums de bovins infestés par Cysticercus bovis ont donné des réactions posi- tives au test du latex, avec des titres agglutinants variables: $1 / 16,1 / 32,1 / 64$ pour $71 \mathrm{p}$. 100 d'entre eux.

3.2. 10 des animaux ont de faibles titres: $1 / 2,1 / 4,1 / 8$.

3.3. 19 p. 100 ont des taux élevés d'anticorps (10 zébus sur un total de 56). Parmi ceux-ci, un tiers ( 3 sur 10) présentaient une cysticercose généralisée, comme on peut le voir dans le tableau $n^{\circ} I$.

Ceci s'explique par le fait que, dans une proportion de 60 à 68 p. 100 , les animaux âgés de plus de trente mois sont généralement immunisés contre la cysticercose ou naturellement résistants.

Le tableau $n^{\circ}$ I montre qu'il existe une certaine corrélation entre les titres agglutinants, les localisations et le nombre de vésicules relevées à l'autopsie.

3.4. Les sérums de bovins polyparasités, mais sans cysticerques, ont également donné des réactions positives, mais à des taux compris entre le $1 / 2$ et le $1 / 16$.

3.5. Les sérums de lapins hyperimmunisés donnent des titres agglutinants de $1 / 512$ et $1 / 1.024$.

En conclusion, seuls, les sérums de haute réactivité sont susceptibles de fournir la preuve formelle de l'existence de la maladie.

3.6. La réaction d'agglutination du latex a été utilisée à plusieurs reprises chez des bovins européens atteints de cysticercose. Les résultats obtenus en Russie par SOKOLOVSKAYA (1966) (19), KOMINSKOV et PHILIPPOV (1967 et 1971) (8), LEIKINA et collab. (1966) (10) et, en Allemagne, par GROSSKLAUS et WALTHER (1970) (6), sont à peu près semblables à ceux du Tchad: le pourcentage d'animaux reconnus porteurs oscille entre 91 et 96 p. 100. Les taux d'agglutination sont de $1 / 16$ chez les veaux et de $1 / 32$ à $1 / 64$ chez les adultes.

Les auteurs russes signalent de fausses réactions positives dans 23 p. 100 des cas, ce que confirment également SƯSSE et FRICK (1970) (20).

\section{Hémagglutination passive :}

- Les immuns sérums donnent des titres agglutinants au $1 / 5000$ et $1 / 10000$. 
TABLEAU N ${ }^{6}$ I

Corrélation entre le nombre de cysticerques et les titres agglutinants dans la reaction d'agglutination du latex

\begin{tabular}{|c|c|c|c|}
\hline \multirow{2}{*}{ Localisations } & \multicolumn{2}{|c|}{ Nombre de Cysticerques } & \multirow{2}{*}{ Titres agglutinants } \\
\hline & Vivants & Calcifiês & \\
\hline $\begin{array}{l}\text { Epaule } \\
\text { " } \\
\text { " } \\
" \\
" \\
\text { " } \\
\text { " }\end{array}$ & $\begin{array}{l}1 \\
1 \\
1 \\
1 \\
2 \\
3\end{array}$ & $\begin{array}{l}3 \\
2 \\
1\end{array}$ & $\begin{array}{l}1 / 8 \\
1 / 8 \\
1 / 32 \\
1 / 32 \\
1 / 16 \\
1 / 32 \\
1 / 16 \\
1 / 64 \\
1 / 64\end{array}$ \\
\hline $\begin{array}{l}\text { Epaule et quartier } \\
\text { antêrieur }\end{array}$ & & 3 & $1 / 64$ \\
\hline $\begin{array}{l}\text { Quartier posterieur } \\
\text { " } \\
\text { " } \\
" \\
" \\
\text { " }\end{array}$ & $\begin{array}{l}1 \\
1 \\
2 \\
2 \\
3\end{array}$ & $\begin{array}{l}1 \\
1 \\
1\end{array}$ & $\begin{array}{l}1 / 32 \\
1 / 12 B \\
1 / 64 \\
1 / 64 \\
1 / 12 B \\
1 / 64 \\
1 / 128 \\
1 / 128\end{array}$ \\
\hline Coeur & 1 & & $1 / 16$ \\
\hline Langue & & 2 & $1 / 32$ \\
\hline $\begin{array}{l}\text { Gênéralisêe } \\
\text { " } \\
" 1 \\
" 1 \\
" \\
"\end{array}$ & $\begin{array}{r}30 \\
25 \\
150 \\
12 \\
13 \\
\\
16 \\
4\end{array}$ & 13 & $\begin{array}{l}1 / 16 \\
1 / 32 \\
1 / 32 \\
1 / 64 \\
1 / 64 \\
1 / 128 \text { et } \\
1 / 256 \\
1 / 128 \\
1 / 256\end{array}$ \\
\hline
\end{tabular}

- Les sérums de bovins, porteurs de vésicules ladres, testés avec l'antigène délipidé de Cysticercus bovis donnent des titres élevés du $1 / 10$ au $1 / 1280$.

La sensibilité de la réaction d'hémagglutination se révèle supérieure à celle du latex, mais la spécificité n'est pas absolue.

- Ces résultats corroborent en grande partie ceux de MOSIMA (1965) (13), de PROCTOR et collab. (1966) (17) et d'ALFEROVA (1969) (1): chez des veaux de 30 à 40 jours, la cysticercose peut être mise en évidence trois semaines après l'infestation initiale (par 2000 oncosphères de Taenia saginata). Vers le trente et unième jour, les taux d'anticorps baissent, puis remontent entre 81 et 101 jours, pour décroître à nouveau vers le quatrième mois.

Par ailleurs, d'après FRICK et SUSSE (1970), la méthode ne permet pas de déceler plus de 42 p. 100 d'animaux ladres. Les fausses réactions positives sont peu nombreuses (8 p. 100).

\section{CONCLUSIONS}

Les réactions croisées sont un obstacle au diagnostic sérologique de la cysticercose bovine, en raison du polyparasitisme qui sévit chez les zébus des zones tropicales.

Ce diagnostic n'offre, dans les limites des méthodes actuellement employées au Laboratoire de Farcha, aucune garantie de spécifícité.

Les techniques utilisées ne permettent qu'une certaine présomption de la maladie.

L'agglutination du latex et l'hémagglutination passive sont les méthodes qui ont donné les résultats les plus intéressants quant à la sensibilité et à la spécificité de la réaction. 


\section{REMERCIEMENTS}

Je remercie tous ceux qui m'ont permis de réaliser ce travail, en particulier Monsieur Que- val, Biologiste du Service de Virologie du Laboratoire de Farcha, Fort-Lamy, pour sa précieuse collaboration et l'aide qu'il m'a apportée dans la conduite de cette recherche.

\section{SUMMARY}

\section{The bovine cysticercosis in Chad. - Serological diagnosis trials}

In Chad, serological diagnosis of bovine cysticercosis is difficult, because, in zebu cattle, the presence of abundant polyparasitism gives often cross reactions.

Generally, the methods lack for specificity. The latex test and the hemagglutination test are the best.

\section{RESUMEN}

\section{La cisticercosis de los bovinos en Chad Ensayo de diagnóstica serologico}

En Chad, el diagnóstico serologico de la cysticercosis de los bovinos es dificil, a causa de reacciones cruzadas que la existencia de un poliparasitismo abundante acarrea en el cebú.

Los métodos carecen, generalmente, de especificidad. La aglutinación del latex y la hemaglutinación pasiva son los mejores.

\section{BIBLIOGRAPHIE}

1. ALFEROVA (V.M.), The indirect haemagglutination test in cysticercosis of cattle. Medskaya Parasit., 1969, 38 (2) : 162-166 (en russe).

2. CAPRON (A.), BIGUET (J.), VERNES (A.), AFCHAIN (D.), Structure antigénique des helminthes. Aspects immunologiques des relations hôte-parasite, Path. Biol., 1968, 16 (3/4) : 121.

3. EUZEBY (J.), Immunologie des helminthoses, Rev. Méd. prér., 1965, 116 (6): 435.

4. FRICK (W.), SÜSSE (H. J ), Immunobiological detection of bovine cysticercosis, Arch. exp. Vet. Med., 1970, 24 : 451-457.

5. GRABER (M.), THOME (M.), La Cysticercose bovine en République du Tchad. Quelques réflexions sur la situation présente, l'étiologie, l'immunité et le traitement de cette zoonose, Rev. Elev. Méd. vét. Pays trop., 1964, 17 (3) : 441-467.

6. GROSSKLAUS (D.), WALTHER (M.), Zur serodiagnose der Zystizerkose des Rindes, Zentbl. Vet. Med., 1970, 17 (8) : 828-839.

7. KOSMINKOV (N. E.), PHILIPPOV (V. V.), Use of polystyrène latex in the diagnosis of cysticercosis in living cattle, Dokl, vses. Akad. sel.-Khoz. Nauk., 1967 (5) : 37-38 (en russe).

8. KOSMINKOV (N. E.), PHILIPPOV (V. V.), Practice of intravital diagnosis of cysticercosis in Bos taurus, 19th Cong. Mond. Méd. vet., Mexico, 1971, 2, 660.

9. LAMINA (J.), HEIN (B.), Untersuchungen zur Frage des immunologischen Nachweiss einer Zystizerkose am lebenden Tier. II. Mitteilung: Die Komplementbindungsreaktion, $D t$. tierärztl Wschr., 1970, 77 : 273-278.

10. LEIKINA (E. S.), SOKOLOVSKAYA (M. O.), POLETAEVA (O. G.), ASTAKHOVA (O. O.), MOSKVIN (S. N.), Immunodiagnosis of bovine cysticercosis and the technique of assessing results obtained, Medskaya Parasit., 1966, 35 (2): 157164 (en russe).

11. MARTIN (C.), La cysticercose bovine au Tchad. Essai de diagnostic sérologique, Thèse Méd. vét., Paris, 1971, 75 p.

12. MELCHER (L. R.), CAMPBELL (D. H.), A serologically active polysaccharide from Trichunella spiralis, Science, N.Y., 1942, 96 (2497): 431.

13. MOSIMA (S. K.), Immunological methods for diagnosing experimental cysticercosis in cattle, Uchen. Zap. Ka.san vet. Inst., 1965, 94, 123-126 (en russe).

14. PAUTRIZEL (R.), BAILENGER (J.), CAILLAU (M.), La distomatose. II. Diagnostic sérologique, Rev. Hyg. Méd. soc., 1960, 7: 617.

15. POWELL (S. J.), PROCTOR (E. M.), HARNETT (W.), Neurological complications of cysticercosis in Africans : a clinical and serological study, Ann. trop. Med. Parasit., 1966, 60 (2) : 159.

16. POWELL (S. J.), PROCTOR (E. M.), WILMOT, MAC LEOD, cysticercosis and epilepsy in Africans, a clinical and serological study, Ann. trop. Med. Parasit., 1966, 60 (2): 152.

17. PROCTOR (E. M.), POWELL (S. J.), ELSONDEW (R.), The serological diagnosis of cysticercosis, Ann. trop. Med. Parasit., 1966, 60 (2): 146-151.

18. SINGER (J. M.), PLOTZ (C. M.), The latex fixation test: application to the serologic diagnosis of rheumatoid arthritis, Am. J. Med., 1956, 21 : 888 .

19. SOKOLOVSKAYA (O. M), Experimental study of serum agglutination test for Bovine cysticercosis, Mater. Konf. vses. Obshch. Gel 'mint, Moscou, 1966, 2 : 175-83 (en russe).

20. SUSSE (H. J.), FRICK (W.), The serological diagnosis of cysticercosis in cattle, Mh. Vet. Med., $1970,25: 435-438$. 